\title{
Evidências científicas sobre a qualidade de vida dos pacientes com insuficiência cardíaca: revisão integrativa*
}

\author{
Scientific evidence about quality of life of patients with heart \\ failure: integrative review
}

Flávia Ribeiro do Carmo ${ }^{1}$ - Harriet Bárbara Maruxo² • Willian Alves dos Santos ${ }^{3}$

\begin{abstract}
RESUMO
Objetiva-se identificar evidências científicas sobre a qualidade de vida em pacientes diagnosticados com insuficiência cardíaca. Revisão integrativa realizada nas bases de dados Literatura Latino-Americana e do Caribe em Ciências da Saúde (LILACS), Base de Dados de Enfermagem (BDENF) e Sistema Online de Busca e Análise de Literatura Médica (MEDLINE) com a seguinte questão norteadora: Como a insuficiência cardíaca interfere na qualidade de vida dos pacientes cardiológicos? Foi utilizada a estratégia PICO com recorte temporal de 2011 a 2016, nos idiomas português, inglês e espanhol. As informações foram tabuladas em programa Microsoft Excel ${ }^{\circledR}$ e processadas a partir de estratégia: média aritmética descritiva simples, frequência absoluta e relativa. Encontrou-se 712 artigos na sua totalidade, após aplicação dos critérios de inclusão e exclusão foram selecionados 17 artigos científicos avaliados a partir da categoria: os principais fatores que comprometem a qualidade de vida dos pacientes com insuficiência cardíaca. Conclui-se que os pacientes com insuficiência cardíaca apresentam intolerância à realização de atividades físicas e têm seu sono prejudicado por conta da dispneia e fadiga, sintomas que contribuem para o declínio da qualidade de vida.
\end{abstract}

Palavras-chave: Insuficiência Cardíaca; Qualidade de Vida; Enfermagem.

\section{ABSTRACT}

The aim is to identify scientific evidence on the quality of life in patients diagnosed with heart failure. Integrative review carried out in the Literatura Latino-Americana e do Caribe em Ciências da Saúde (LILACS), Base de Dados de Enfermagem (BDENF) e Sistema Online de Busca e Análise de Literatura Médica (MEDLINE) databases with the question: How does heart failure interfere with the quality of life of cardiology patients? The PICO strategy with time cut from 2011 to 2016 was used in Portuguese, English and Spanish. The information was tabulated in Microsoft Excel ${ }^{\circledR}$ program and processed from strategy: simple descriptive arithmetic mean, absolute and relative frequency. It found 712 articles in their totality, after applying the inclusion and exclusion criteria, were selected 17 scientific articles evaluated from the category: the main factors that compromise the quality of life of patients with heart failure. It concludes that the patients with heart failure are intolerant to physical activities and sleep impaired due to dyspnea and fatigue, such symptoms that contribute to the decline of quality of life.

Keywords: Heart failure; Quality of life; Nursing.

${ }^{1}$ Enfermeira. Pós-graduanda em Enfermagem Cardiológica, Universidade Estácio de Sá (UNESA). Rio de Janeiro (RJ), Brasil. E-mail: flaflanit@bol.com.br

${ }^{2}$ Enfermeira. Mestre em Ciências da Saúde, Escola de Enfermagem da Universidade de São Paulo (USP). Professora do curso de graduação em Enfermagem da Universidade CIDADE de São Paulo (UNICID). Especialista em enfermagem cardiovascular pelo Instituto Dante Pazzanese de Cardiologia. E-mail: harribabi@gmail.com;

${ }^{3}$ Enfermeiro, Mestre em Ciências do Cuidado em Saúde pela Escola de Enfermagem Aurora de Afonso Costa da Universidade Federal Fluminense (EEAAC/UFF). Niterói (RJ), Brasil. E-mail: willian.allves@hotmail.com;

Endereço para correspondência: Flávia Ribeiro do Carmo. Travessa São Luis, 751, Largo da Batalha, Pendotiba. Niterói (RJ), Brasil. CEP: 24310-360. E-mail: flaflanit@bol.com *Manuscrito extraído de trabalho de conclusão de curso (especialização) intitulada: Qualidade de vida dos pacientes com insuficiência cardíaca: uma revisão integrativa. Universidade Estácio de Sá. 2016.

Os autores declaram não haver conflito de interesse. 


\section{INTRODUÇÃO}

Nos dias atuais, a saúde associada à qualidade de vida vem sendo muito discutida, principalmente na população que apresenta doenças cardiovasculares, uma vez que tais patologias ocasionam um número significativo de mortes e mudanças em seus estilos de vida. No Brasil, há cerca de 6,5 milhões de pacientes com insuficiência cardíaca prevalente após os 60 anos de idade, sendo considerada problema de saúde pública. Apesar dos inúmeros avanços tecnológicos para o seu diagnóstico a mortalidade e morbidade continuam altas e os índices apontam que cerca de $30 \%$ são reinternados em um intervalo de tempo de um ano piorando seu desempenho físico, emocional e contribuindo para diminuição da qualidade de vida ${ }^{1-4}$.

A insuficiência cardíaca caracteriza-se por ser uma síndrome sistêmica que promove inadequada capacidade do coração de bombear sangue para atender as demandas de oxigênio e nutrientes dos tecidos ${ }^{5-6}$. 0 surgimento dessa patologia pode ocorrer devido à um distúrbio na contração muscular gerando uma anormalidade primária do músculo cardíaco, como ocorre na miocardiopatia e miocardite virótica. Ela ainda pode surgir por uma aterosclerose coronariana que causa isquemia e infarto do miocárdio ${ }^{7}$.

Para mensurar o grau de acometimento do músculo cardíaco, quanto à intensidade dos sintomas dos pacientes com insuficiência cardíaca a New York Heart Association (NYHA) propôs quatro classes: Classe 1 (apresenta nenhuma limitação nas atividades cotidianas, ausência dos sintomas); Classe 2 (sintomas desencadeados por atividades cotidianas); Classe 3 (sintomas desencadeados em atividades cotidianas ou pequenos esforços); Classe 4 (sintomas da insuficiência cardíaca em repouso) ${ }^{8}$.

A presença de sinais e sintomas proporciona, também, um impacto na fonte de renda de seu portador, uma vez que suas atividades físicas ficam limitadas, incapacitando-o para o exercício da profissão e submetendo-o a depender do auxílio doença9 .

Com base nisso, observa-se que a progressão desta síndrome promove diversas dificuldades na funcionalidade e na autonomia desses pacientes levando-os às limitações na sua rotina diária. Assim, faz-se relevante desenvolver um maior conhecimento a respeito da qualidade de vida desses pacientes. Para a Organização Mundial da Saúde, qualidade de vida trata-se da percepção que o indivíduo tem a sua volta, em relação aos seus objetivos, expectativas, padrões e preocupações. Possuir qualidade de vida não significa somente ter saúde física e mental, mas que o indivíduo tenha capacidade de reagir de forma positiva aos problemas que o cercam ${ }^{10}$.

De acordo com esses esclarecimentos sobre a qualidade de vida, as funções físicas, psicológicas e sociais desses pacientes que podem ser influenciadas pela doença, ou seja, sua habilidade ou capacidade de readaptação à sua nova condição pode afetar negativamente a sua percepção de vida. Dessa forma, tal pesquisa teve como objetivo: identificar evidências científicas sobre a qualidade de vida em pacientes diagnosticados com insuficiência cardíaca.

\section{MÉTODO}

Trata-se de uma revisão integrativa da literatura. Tal método é implementado com a finalidade de sintetizar, de modo abrangente, resultados de pesquisas de diversas metodologias, fornecendo informações amplas sobre determinado tema ${ }^{11}$.

A pesquisa em questão foi construída em 6 etapas: elaboração da pergunta norteadora; busca ou amostragem na literatura; coleta de dados; análise crítica dos estudos incluídos; discussão dos resultados e apresentação da revisão integrativa ${ }^{11}$ a partir do levantamento bibliográfico eletrônico nas seguintes bases de dados via Biblioteca Virtual de Saúde (BVS): Literatura Latino Americana e do Caribe em Ciências da Saúde (LILACS), e Base de Dados Brasileiras de Enfermagem (BDENF) e via PubMed: Medical Literature Analysis and Retrieval Sistem on-line (MEDLINE) norteando-se pela seguinte questão: Como a insuficiência cardíaca interfere na qualidade de vida dos pacientes cardiológicos?

Para elaboração da questão norteadora foi utilizada a estratégia $\mathrm{PICO}^{12}$, que representa o acrômio para $(\mathrm{P}=$ Paciente ou Problema, I = Intervenção, C = Comparação ou controle, $\mathrm{O}=$ Outcomes ou desfechos). Ressalta-se que neste estudo foi considerado apenas $P=$ pacientes cardiológicos, I = insuficiência cardíaca e $\mathrm{O}$ = qualidade de vida (PIO).

A estratégia de busca nas bases de dados foi executada por meio do cruzamento dos Descritores em Ciências da Saúde (DeCs): Insuficiência cardíaca, qualidade de vida e enfermagem e por Medical Subject Headings Mesh Terms: Heart Failure, Quality of Life and Nurses. O cruzamento entre os descritores foi conectado pelo o operador booleano AND.

As etapas para seleção dos artigos científicos realizaram-se a partir da adoção dos critérios de elegibilidade. Os critérios de inclusão estabelecidos foram: artigos científicos com textos completos disponíveis online, nos idiomas português, inglês e espanhol, com recorte temporal de 2011 à 2016, apresentando desenhos de cunho experimental, quase experimental, observacional e de revisão, com aderência ao eixo temático.

Foram excluídas as publicações que não atendiam ao objetivo desta pesquisa, que apresentaram estudos realizados com animais, crianças, adolescentes e que se surgiram simultaneamente nos cruzamentos em bases de dados.

Após a seleção das evidências científicas, executou-se a leitura e análise minuciosa dos textos. Para viabilizar a identificação das evidências científicas, realizou-se a caracterização dos estudos segundo o país de pesquisa, periódico, base de dados indexadora, ano de publicação, 
tipo de estudo e nível de evidência por meio da proposta de Melnyk e Fineout-Overholt ${ }^{13}$.

As informações foram tabuladas no programa Microsoft Excel $^{\oplus}$ e processadas a partir da estratégia de medida de tendência central com a média aritmética descritiva simples, frequência absoluta e relativa. Utilizou-se o recurso ilustrativo de quadros e figuras.

\section{RESULTADOS}

Foram identificados 712 artigos em sua totalidade.Após os critérios de exclusão, inclusão, leitura prévia de títulos e resumos foram incluídos 17 artigos, lidos na íntegra, como pode ser observado na figura 1.

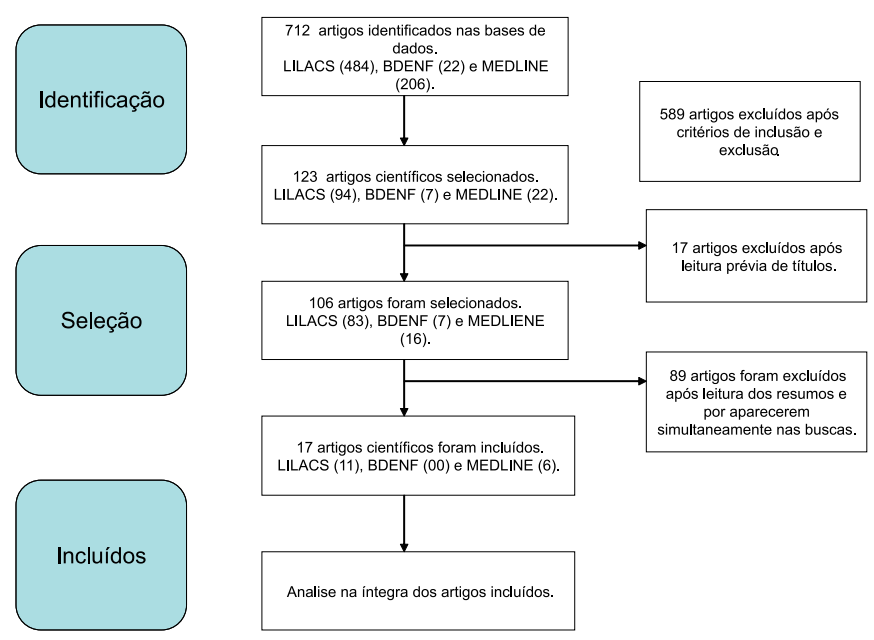

Figura 1: Fluxograma esquemático da estratégia da busca dos artigos. Niterói, RJ, Brasil, 2016.

Fonte: dados da própria pesquisa.

Após a leitura dos artigos selecionou-se um total de 17 trabalhos que atenderam aos critérios de inclusão, constituindo a amostra final desse estudo.

Em relação aos anos de publicação, foi possível observar que houve maior publicação no ano de 2013 e 2015 ambos com (23,53\%), seguido de 2011, 2012 e 2014, todos com $(17,65 \%)$ cada. No que tange a metodologia, foi possível identificar que houve maior predominância de estudo de cunho transversal $(58,82 \%)$ seguido de ensaio clínico (23,53\%). Metodologias de revisão de literatura, descritiva e estudo de caso foram observadas, comportando $(5,88 \%)$ cada.

Além disso, o país que mais publicou sobre o tema foi o Brasil (41,18\%), seguido dos Estados Unidos (23,53\%).

Já em relação às bases de dados, houve predomínio de artigos indexados na Lilacs (64,71\%) e Medline (35,29\%). 0 nível de evidência também foi avaliado, o que demonstrou que (70,59\%) são de nível VI, (23,53\%) II e (5,88\%) V.

As evidências científicas foram caracterizadas conforme o título, referência, país de pesquisa, o periódico, base de dados e ano, método utilizado na pesquisa e nível de evidência, como mostrado no quadro 1.
Quadro 1. Caracterização dos estudos selecionados. Niterói, RJ, Brasil, 2016.

\begin{tabular}{|c|c|c|}
\hline VARIÁVEIS & $\mathbf{N}$ & $\%$ \\
\hline \multicolumn{3}{|l|}{ Nacionalidade } \\
\hline Brasil & 7 & 41,18 \\
\hline EUA & 4 & 23,53 \\
\hline Chile & 1 & 5,88 \\
\hline Cuba & 1 & 5,88 \\
\hline Grécia & 1 & 5,88 \\
\hline Iran & 1 & 5,88 \\
\hline Dinamarca & 1 & 5,88 \\
\hline Japão & 1 & 5,88 \\
\hline Total & 17 & 100,00 \\
\hline \multicolumn{3}{|l|}{ Base de dados } \\
\hline Lilacs & 11 & 64,71 \\
\hline Medline & 6 & 35,29 \\
\hline Total & 17 & 100,00 \\
\hline \multicolumn{3}{|l|}{ Ano de publicação } \\
\hline 2011 & 3 & 17,65 \\
\hline 2012 & 3 & 17,65 \\
\hline 2013 & 4 & 23,53 \\
\hline 2014 & 3 & 17,65 \\
\hline 2015 & 4 & 23,53 \\
\hline Total & 17 & 100,00 \\
\hline \multicolumn{3}{|l|}{ Metodologia } \\
\hline Transversal & 10 & 58,82 \\
\hline Ensaio clínico & 4 & 23,53 \\
\hline Revisão de literatura & 1 & 5,88 \\
\hline Estudo de caso & 1 & 5,88 \\
\hline Descritivo & 1 & 5,88 \\
\hline Total & 17 & 100,00 \\
\hline \multicolumn{3}{|l|}{ Nível de evidência } \\
\hline II & 4 & 23,53 \\
\hline V & 1 & 5,88 \\
\hline VI & 12 & 70,59 \\
\hline Total & 17 & 100,00 \\
\hline
\end{tabular}

Fonte: dados da própria pesquisa.

Tendo em vista a análise profunda dos artigos que compõem o acervo amostral, foi possível identificar que há determinados sintomas que acometem o paciente com insuficiência cardíaca. Desse modo, o quadro 3 mostra os principais fatores que interferem na qualidade de vida desses pacientes. 
Quadro 2. Distribuição dos estudos incluídos na revisão integrativa segundo nível de evidência. Niterói, RJ, Brasil, 2016.

\begin{tabular}{|c|c|c|c|c|c|c|}
\hline $\mathrm{N}^{\circ}$ & Referência & $\begin{array}{l}\text { País de } \\
\text { pesquisa }\end{array}$ & Periódico & Base de Dados/Ano & Tipo de estudo & $\begin{array}{l}\text { Nível de } \\
\text { evidência }\end{array}$ \\
\hline A1 & AGREN, et al.,2013. & Dinamarca & J Clin Nurs & Medline 2013 & Ensaio clínico & II \\
\hline A2 & $\begin{array}{l}\text { BARBOSA, et } \\
\text { al.,2014. }\end{array}$ & Brasil & $\begin{array}{c}\text { Revista Brasileira } \\
\text { de Cardiologia }\end{array}$ & Lilacs 2014 & Transversal & $\mathrm{VI}$ \\
\hline$A 3$ & $\begin{array}{l}\text { DEKKER, et } \\
\text { al.,2014. }\end{array}$ & EUA & $\begin{array}{c}\text { Eur J Cardiovasc } \\
\text { Nurs }\end{array}$ & Lilacs 2014 & Transversal & VI \\
\hline A4 & $\begin{array}{l}\text { FORRELAT, et } \\
\text { al.,2012. }\end{array}$ & Cuba & $\begin{array}{l}\text { Rev. Cuba. Hematol. } \\
\text { Inmunol. Hemoter }\end{array}$ & Lilacs 2012 & Transversal & VI \\
\hline A5 & $\begin{array}{l}\text { GONZÁLEZ, } \\
\text { Marcela; } \\
\text { PEDREIRO, } \\
\text { Victor.,2013. }\end{array}$ & Chile & $\begin{array}{l}\text { Revista Chilena de } \\
\text { Cardiologia }\end{array}$ & Lilacs 2013 & $\begin{array}{l}\text { Revisão de } \\
\text { literatura }\end{array}$ & V \\
\hline A6 & $\begin{array}{l}\text { GUEDES, et } \\
\text { al.,2012. }\end{array}$ & Brasil & $\begin{array}{l}\text { Revista Lat. Am. da } \\
\text { enfermagem }\end{array}$ & Lilacs 2012 & Transversal & $\mathrm{VI}$ \\
\hline A7 & $\begin{array}{l}\text { HEO, et al., } \\
2012 .\end{array}$ & EUA & $\begin{array}{c}\text { Eur J Cardiovasc } \\
\text { Nurs }\end{array}$ & Lilacs 2012 & Transversal & $\mathrm{VI}$ \\
\hline A8 & HICKEY, et al.,2013. & EUA & J Clin Nurs & Medline 2013 & Transversal & VI \\
\hline A9 & LAMARCA., 2015. & Brasil & $\begin{array}{c}\text { Banco de teses } \\
\text { UERJ }\end{array}$ & Lilacs 2015 & Transversal & $\mathrm{VI}$ \\
\hline A10 & $\begin{array}{l}\text { MATSUO, et } \\
\text { al.,2013. }\end{array}$ & Japão & Circulation Journal & Medline 2013 & Ensaio clínico & II \\
\hline A11 & $\begin{array}{l}\text { MEHRALIA, et } \\
\text { al.,2014. }\end{array}$ & Iran & $\begin{array}{l}\text { Global Journal of } \\
\text { Health Science }\end{array}$ & Medline 2014 & Ensaio clínico & II \\
\hline $\mathrm{A} 12$ & $\begin{array}{l}\text { MERAVIGLIA, et } \\
\text { al.,2015. }\end{array}$ & EUA & J Cardiovasc. Nurs & Medline 2015 & Ensaio clínico & II \\
\hline $\mathrm{A} 13$ & $\begin{array}{l}\text { MICHALIS, et } \\
\text { al.,2015. }\end{array}$ & Grécia & Hellenic J. Cardiol & Medline 2015 & Transversal & VI \\
\hline $\mathrm{A} 14$ & NASO, et al.,2011. & Brasil & $\begin{array}{c}\text { Fisioterapia e } \\
\text { pesquisa }\end{array}$ & Lilacs 2011 & Transversal & $\mathrm{VI}$ \\
\hline A15 & $\begin{array}{l}\text { SANTOS, et } \\
\text { al.,2011. }\end{array}$ & Brasil & $\begin{array}{l}\text { Revista Brasileira } \\
\text { de enfermagem }\end{array}$ & Lilacs 2011 & Descritivo & $\mathrm{VI}$ \\
\hline A16 & $\begin{array}{l}\text { SILVA; RABELO; DA } \\
\text { SILVA., } 2015 .\end{array}$ & Brasil & Revista Aquichan & Lilacs 2015 & Estudo de caso & VI \\
\hline A17 & $\begin{array}{l}\text { VALADARES, et } \\
\text { al.,2011. }\end{array}$ & Brasil & $\begin{array}{l}\text { Rev. Bras. Med. } \\
\text { Esporte }\end{array}$ & Lilacs 2011 & Transversal & VI \\
\hline
\end{tabular}

Fonte: dados da pesquisa dos autores.

Quadro 3. Principais sinais e sintomas que comprometem a qualidade de vida dos pacientes com insuficiência cardíaca. Niterói, RJ, Brasil, 2016.

\begin{tabular}{|l|}
\hline $\begin{array}{l}\text { Principais sinais e sintomas que interferem na qualidade de } \\
\text { vida dos pacientes com insuficiência cardíaca. }\end{array}$ \\
\hline - Fadiga \\
- Dispnéia \\
- Tosse \\
- Edema \\
- Náusea \\
- Dor \\
- Ortopneia \\
- Palpitação
\end{tabular}

Fonte: dados da própria pesquisa.

\section{DISCUSSÃO}

Os resultados obtidos por meio deste estudo foram organizados em uma categoria para discussão dos resultados: os principais fatores que comprometem a qualidade de vida dos pacientes com insuficiência cardíaca.

\section{Categoria 1: Os principais fatores que comprometem a qualidade de vida dos pacientes com insuficiência cardíaca}

Geralmente os pacientes que apresentam problemas cardíacos sofrem modificação no padrão de vida, determinado pela incapacidade de executar atividades cotidianas. No que tange aos pacientes com insuficiência cardíaca, os sintomas são os principais fatores que interferem negativamente 
na qualidade de vida, destacando-se, dentre eles: dor ou desconforto precordial, dispneia, ortopneia, palpitação, síncope, fadiga e edema. Dessa forma, torna-se importante à atuação da enfermagem a fim de proporcionar a minimização dos sintomas físicos e psicológicos, promovendo assim, a qualidade de vida desses pacientes ${ }^{14}$.

Em um estudo, observou-se que a insuficiência cardíaca tem forte impacto na vida do paciente, principalmente decorrente das limitações impostas por sintomas físicos e psicológicos, entre os sintomas físicos destacam-se: fadiga e dispneia e, já entre os sintomas emocionais, destacam-se o medo, a insegurança e a tristeza. Há, também, a dificuldade em conviver com as alterações da doença cardíaca, devido à mudança do cotidiano e aos sentimentos ameaçadores que emergem em virtude às restrições submetidas ${ }^{15}$.

No que tange à fadiga, principal sintoma relacionada à deterioração da qualidade de vida, observa-se que tal sintoma é um fator associado às limitações para a conservação de um estilo de vida desejável de autonomia e independência ${ }^{16-17}$. Nos resultados do estudo em questão foi possível observar que tal sintoma é o principal fator que compromete a qualidade de vida dos pacientes com essa patologia, necessitando de medidas de integração interdisciplinar para o seu controle.

Em estudo transversal realizado com 10 pacientes diagnosticados com insuficiência cardíaca com classe funcional III e IV de ambos os sexos, destacou que quanto maior a progressão da doença e a função cardíaca, maior é o comprometimento da capacidade funcional, o que interfere diretamente no desempenho das atividades físicas. Esse dado é resultado da utilização do questionário Short Form Health Survery (SF-36) e as variáveis cardiopulmonares, como o teste de caminhada de 6 minutos (TC6M), avaliando os esforços e a dispneia presente ${ }^{18}$.

Geralmente é observada intolerância à atividade nos pacientes com doenças cardíacas. A impossibilidade de realizar atividades físicas contribui para um estado ruim de qualidade de vida, principalmente, na população idosa ${ }^{19}$. Ainda, em um estudo transversal realizado com 63 pacientes idosos com classe II e IV da NYHA, o estado geral de saúde foi baixo e estava correlacionado com: gravidade da fadiga em repouso, intolerância aos exercícios, tonturas, palpitações e dor no peito ${ }^{20}$. Em um estudo realizado com 66 pacientes que avaliou a correlação entre padrões pulmonares, capacidade funcional e a classe NYHA determinou que pacientes com insuficiência cardíaca apresentam diminuição da condição pulmonar, capacidade físico-funcional e qualidade de vida relacionada ao aumento da classe funcional da NYHA. Isso mostra que, quanto maior o índice da classe NYHA, maior a debilidade de vida do paciente com insuficiência cardíaca ${ }^{21}$.

A insuficiência cardíaca proporciona limitações sociais que interferem negativamente na qualidade de vida dos indivíduos devido à ocorrência de quadros agudos que favorecem a hospitalização recorrente e mudança no estilo de vida ${ }^{15}$.
Outro aspecto relevante a ser mencionado é o fato de a patologia interferir diretamente no padrão do sono. Dentre os principais fatores que estão associados à dificuldade para dormir, inerentes a insuficiência cardíaca, destacamse: a nictúria, a interrupção do sono durante à noite e a dificuldade respiratória. Essa deficiência na qualidade do sono pode levar ao déficit da qualidade de vida do paciente com insuficiência cardíaca ${ }^{17}$.

Em relação ao sexo, um estudo transversal, unicêntrico e prospectivo buscou avaliar tal associação com a qualidade de vida e insuficiência cardíaca. Como resultado, obteve-se que, apesar do menor número de internações hospitalares, observou-se uma pior qualidade de vida nos pacientes do sexo feminino em comparação ao masculino ${ }^{22}$. Sugere-se que, diante dos resultados, a população feminina apresenta maior dificuldade em lidar com as situações ocorridas decorrentes das complicações da doença, sofrendo um mal-estar físico e psíquico, resultando em uma piora da qualidade de vida.

Além disso, um estudo internacional demonstrou que há relação entre o estado de saúde e resultados sociais em pacientes com insuficiência cardíaca. As percepções de qualidade do suporte social e status econômico dos pacientes foram relacionados com o questionário Minnesota

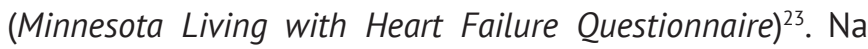
análise do estudo observou-se que a população com baixa renda teve maior índice de hospitalizações, mesmo após a abordagem de diferentes linhas de tratamento.

A mudança do estilo de vida e a cronicidade da doença culminam no desenvolvimento de depressão e ansiedade. Corroborando com isso, um estudo com 556 pacientes demonstrou que um terço deles tinha sintomas como: depressão e ansiedade ou ambos simultaneamente. Houve uma relação dose-resposta entre sintomas depressivos e sintomas de ansiedade e, níveis mais elevados de sintomas depressivos foram associados com um nível mais elevado de sintomas de ansiedade ${ }^{24}$.

Ainda sobre aspectos psicológicos no que tange a depressão, um estudo realizado com 190 pacientes internados com insuficiência cardíaca registrou que o nível de depressão tinha correlação com a progressão da doença, atribuindo como uma barreira ao tratamento e uma piora na qualidade de vida. Evidenciou que pacientes com classe IV da NYHA eram mais deprimidos que aqueles com a classe II e $\mathrm{III}^{25}$. Sugere-se, com isso, que pacientes que apresentam maior comprometimento patológico com classe NYHA elevada, a qual o paciente encontrase praticamente impossibilitado de realizar atividades diárias, apresentando maior tendência a se isolar do seu seio social e familiar, isso pode justificar os altos índices de depressão nessa população.

Inúmeras práticas podem ser executadas para promover a qualidade de vida dos pacientes. As principais intervenções que apresentam resultados promissores são: intervenções de educação (reconhecimento dos sinais e sintomas, adesão ao tratamento medicamentoso, mudança 
no estilo de vida e peso diário); aconselhamento por telefone ou vídeo (para definir a terapêutica diurética); e atividade física (teste de caminhada de 6 minutos e aeróbica). A implementação de estratégias de intervenções multidisciplinares tem um efeito positivo sobre o aumento na qualidade de vida ${ }^{26-27}$.

No que concerne à prática de enfermagem, a utilização de teorias do cuidado pode ser uma importante aliada na promoção do conforto desses pacientes. Um estudo que possibilitou a utilização da Teoria do Conforto de Katharine Kolcaba no cenário do cuidado ao paciente com insuficiência cardíaca, e evidenciou que a teoria é de fácil aplicabilidade, proporcionando destacável conforto por meio do cuidado de enfermagem em domicílio ${ }^{28}$.

Ressalta-se a importância do processo de enfermagem no cenário interdisciplinar dirigido à pessoa com insuficiência cardíaca, tendo como fundamento uma teoria de enfermagem. Isso porque a teoria, ao ser implantada, revela-se como um complexo conceitual que solidifica a Enfermagem, "além de se revelar como um modo de ser com o outro, que incentiva a proximidade, a empatia, a interação e o compromisso, buscando sempre a autonomia e valorização do indivíduo"28.

Estudos apontam ainda, que os enfermeiros que prestam assistência aos pacientes com insuficiência cardíaca tem um papel fundamental na avaliação de aplicação de instrumentos para testar sua eficácia e veracidade, além de participar de múltiplos serviços educacionais que visam melhorar à qualidade de vida ${ }^{29-30}$. Com isso, o enfermeiro, como agente multiplicador do cuidado em cardiologia, apresenta função crucial na prevenção,controle e manuseio dos sinais e sintomas em pacientes com insuficiência cardíaca, promovendo, assim, a melhora da qualidade de vida desses pacientes.

\section{CONCLUSÃO}

Evidencia-se que os sinais e sintomas da insuficiência cardíaca são os principais fatores que ocasionam a deterioração da qualidade de pacientes que possuem tal patologia. Dentre eles destacam-se a dispneia, que gera alteração no estilo de vida, intolerância às atividades físicas, bem como mudança no padrão de sono. As palpitações, dor precordial e ortopneia também são fatores que podem contribuir para a ocorrência desse evento.

Com isso, torna-se imprescindível que a enfermagem atue no processo de educação continuada desses pacientes, promovendo o conhecimento à cerca dos sintomas e da importância do balanço hídrico, da adesão terapêutica, controle de peso, bem como promover a execução de atividade física conforme as limitações individuais de cada paciente. Além disso, poder estabelecer diagnósticos de enfermagem de acordo com a CIPE ${ }^{\circledR}$ (Classificação Internacional para a Prática de Enfermagem) e NANDA (Nursing Diagnoses da North American Nursing Diagnosis Association), para assim, promover intervenções de maneira assertiva para a obtenção de resultados promissores.
O conhecimento dos principais fatores que interferem na qualidade de vida de pacientes diagnosticados com insuficiência cardíaca pode influenciar na intervenção e na aplicabilidade de condutas terapêuticas para atingir uma melhora para esse grupo, pois conhecer o perfil desses pacientes facilitaria ações para o controle desta patologia e consequentemente melhora na sua qualidade de vida.

A pesquisa em questão apresentou como limitação a inclusão de estudos com alto poder de evidência, destacando a necessidade de realização de investigações que contenham o ensaio clínico randomizado como método de estudo.

Assim, esperasse que tal estudo contribua para a prática de enfermagem no que se refere a identificação de fatores que comprometem a qualidade de vida do paciente com insuficiência cardíaca, incentivando a realização de outras investigações que abordem o mesmo eixo temático e auxiliando no aperfeiçoamento da prática de enfermagem e no atendimento holístico ao paciente cardiológico.

\section{REFERÊNCIAS}

1. Ferreira VMP, Silva LNO, Furuya RK, Schnidt A, Rossi LA, Dantas RAS.Autocuidado,senso decoerênciaedepressãoempacientes hospitalizados por insuficiência cardíaca descompensada. Rev. Escola de Enf USP [Internet]. 2015 [acesso em 19 de Janeiro de 2016]; 49(3):388-94. Disponível em: http://www. scielo.br/scielo.php?script=sci_arttext \&nrm=iso \&lng= pt\&tlng=pt\&pid=S0080-62342015000300387.

2. Gaui EN, Klein CH, Oliveira GMM. Mortalidade proporcional por insuficiência cardíaca e doenças isquêmicas do coração nas regiões do brasil de 2004 a 2011. Arq Bras Cardiol [Internet]. 2016 [acesso em 20 de Janeiro de 2017]; 107(3):230-8. Disponível em: http://www.arquivosonline. com.br/2016/10703/pdf/10703005.pdf.

3. Gottdiener JS, Arnold AM, Aurigemma GP, Polak JF, Tracy RP, Kitzman DW, et al. Predictors of congestive heart failure in the elderly: the Cardiovascular Health Study. J Am Coll Cardiol [Internet]. 2000 [acesso em 03 de Marco de 2017]; 35(6):1628-37. Disponível em: https://www.ncbi.nlm.nih. gov/pubmed/10807470.

4. Polegato BF, Minicucci MF, Azevedo OS, Gonçalves AF, Lima AF, Martinez PF et al. Associação entre variáveis funcionais e insuficiência cardíaca após o infarto do miocárdio em ratos. Arq Bras Cardiol [Internet]. 2016 [acesso em 20 de Janeiro de 2017]; 106(2):105-12. Disponível em: http://www. arquivosonline.com.br/2016/10602/pdf/10602005.pdf.

5. Amaral LN, Machado RC. Perfil clínico de pacientes com insuficiência cardíaca para embasar a prática clínica do enfermeiro. Revista Brasil [Internet]. 2016 [acesso em 26 de janeiro de 2017]; 15(2):90-7. Disponível em: http:// portalatlanticaeditora.com.br/index.php/enfermagembrasil/ article/view/172/879.

6. Bocchi EA, Marcondes-Braga FG, Bacal F, Ferraz AS, Albuquerque D, Rodrigues $D$ et al. Sociedade Brasileira de Cardiologia.Atualização da Diretriz Brasileira de Insuficiência Cardíaca Crônica - 2012. Arq Bras Cardiol [Internet]. 2012 [acesso em 17 de Janeiro de 2017]; 98(1):1-33. 
Disponível em: http://www.scielo.br/scielo.php?script=sci arttext\&pid=S0066-782X2012000700001.

7. Corrêa LA, Santos I, Rocha RM, Tura BR, Albuquerque DC. Quality of life of patients with hearth failure: a quantitative study. Online Brazilian Journal of Nursing [Internet]. 2009 [acesso em 20 de Janeiro de 2016]; 8(3). Disponível em: http://www.objnursing.uff.br/index.php/nursing/article/ view/j.1676-4285.2009.2518.

8. Bocchi EA, Marcondes-Braga FG, Ayub-Ferreira SM, Rohde LE, Oliveira WA, Almeida DR, et. al. Sociedade Brasileira de Cardiologia. III Diretriz Brasileira de Insuficiência Cardíaca Crônica. Arq Bras Cardiol 2009; 93(1):1-71.

9. Bento VFR, Brofman PRS. Impacto da consulta de enfermagem na frequência de internações em pacientes com insuficiência cardíaca em Curitiba - Paraná. Arquivos Brasileiros de Cardiologia [Internet]. 2009 [Acesso em 20 de Janeiro de 2016]; 92(6):490-969. Disponível em: http:// www.scielo.br/scielo.php?script=sci_arttext \&pid=S0066$782 \times 2009000600013$

10. Soares DA, Toledo JAS, Santos LF, Lima RMB, Galdeano LE. Qualidade de vida de portadores de insuficiência cardíaca. Revista Acta. Paul. Enferm [Internet]. 2008 [acesso em 30 de Janeiro de 2016]; 21(2):243-8. Disponível em: http://www. scielo.br/pdf/ape/v21n2/a02v21n2.pdf .

11. Souza MT, Silva MD, Carvalho R. Revisão integrativa: o que é e como fazer. Einstein. [Internet]. 2010 [acesso em 14 mar 2016]; 8(1 pt 1):102-6. Disponível em: http://www. astresmetodologias.com/material/O_que_e_RIL.pdf.

12. Karino EM, Felli VEA. Enfermagem baseada em evidências: avanços e inovações em revisões sistemáticas. Cienc Cuid Saude [Internet]. 2012 [cited 2017 Jan 26]; 11 (suplem):11-5. Available from: http://eduem.uem.br/ojs/index.php/ CiencCuidSaude/article/view/17048.

13. Cieto BB, Garbuio DC, Carmargo VB de, Napoleão MA. Nursing resources and innovations for hospital discharge: an integrative review. Rev Mineira Enferm [Internet]. 2014 [Acesso em 27 de Janeiro de 2017]; 18(3):752-7. Disponível em: http://www.reme.org.br/artigo/detalhes/960.

14. Lamarca FRRS. Convivendo com a insuficiência cardíaca: uma analise do conhecimento como fator relevante para a qualidade de vida. 2015. 86 f. Dissertação (Mestrado em Enfermagem). Faculdade de Enfermagem, Universidade do Estado do Rio de Janeiro [Internet], RJ, 2015. Disponível em http://pesquisa.bvsalud.org/portal/resource/pt/lil-758241.

15. Santos ACS, Santo FHE, Pestana L, Daher DV, Santana R. Insuficiência cardíaca: estratégias usadas por idosos na busca por qualidade de vida. Revista Brasileira de Enfermagem [Internet]. 2011 [acesso em 29 de junho de 2016]; 64(5):85763. Disponível em: http://pesquisa.bvsalud.org/portal/ resource/pt/lil-618409.

16. Meraviglia M, Clark AP, Mcdougall G, Riegel B, Joiner GR, Innerarity S. Health Status and Self-care Outcomes Following an Education-Support Intervention for People with Chronic Heart Failure. Eur. J. Cardiovasc Nurs [Internet]. 2015 [acesso em 25 de Junho de 2016]; 30(4):3-13. Disponível em: http:// www.ncbi.nlm.nih.gov/pmc/articles/PMC4276559/.

17. Guedes ES, Santos MA, Barbosa RL, Cruz DALM. Sleeping difficulties reported by patients with heart failure. Revista Lat Am Enfermagem [Internet]. 2012 [acesso em 20 de Julho de 2016]; 20(4): 644-50. Disponível em: http://pesquisa. bvsalud.org/portal/resource/pt/lil-649710.

18. Valadares Y, Corrêa KS, Silva BO, Araujo CLP, Karloh M, Mayer AF. Aplicabilidade de testes de atividades de vida diária em indivíduos com insuficiência cardíaca. Revista Brasileira Med. Esporte [Internet]. 2011 [acesso em 20 de Janeiro de 2016]; 17(5):310-4. Disponível em: http://pesquisa.bvsalud.org/ portal/resource/pt/lil-611394.

19. Forrelat MB, Henándes A, Guerrero YM, Dominguez YL, Naranjo AIC, Ramirez P. Importância de los ejercicios físicos para lograr uma ancianidad saludable. Revista Cuba Hematol. Inmunol. Hemoter [Internet]. 2012 [acesso em 17 de Janeiro de 2016]; 28(1):34-40. Disponível em: http://pesquisa. bvsalud.org/portal/resource/pt/lil-628576.

20. Hickey KT, Reiffel J, Sciacca RR,Whang W, Biviano A, Baumeister $M$, Castillo $C$ et al. Correlating perceived arrhythmia symptoms and quality of life in an older population with heart failure: a prospective, single centre, urban clinic study. J. Clin Nurs [Internet]. 2013 [acesso em 20 de Janeiro de 2016]; 22(34):434-44. Disponível em: http://www.ncbi.nlm.nih.gov/ pubmed/23301579.

21. Naso FCD, Pereira JS, Beatricci SZ, Bianchi RG, Dias AS, Monteiro MB. A classe da NYHA tem relação com a condição funcional e qualidade de vida na insuficiência cardíaca. Revista Fisioter. Pesqui [Internet]. 2011 [acesso em 25 de Junho de 2016]; 18(2):157-63. Disponível em: http:// pesquisa.bvsalud.org/portal/resource/pt/lil-610147.

22. Barbosa RR, Franklin RV, Stefenoni AV, Moraes VD, Jacques TM, Serpa RG, Calil AO, Barbosa LMF. Análise da qualidade de vida em homens e mulheres portadores de insuficiência cardíaca. Rev. bras. Cardiol [Internet]. 2014 [Acesso em 20 de Junho de 2016]; 27(3):97-103. Disponível em http://pesquisa. bvsalud.org/portal/resource/pt/lil-719581.

23. Heo S, Moser DK, Chung ML. Social status, health-related quality of life, and event-free survival in patients with heart failure. Eur. J. Cardiovasc Nurs [Internet]. 2012 [acesso em 19 de Junho de 2016]; 11(2):141-9. Disponível em: http:// pesquisa.bvsalud.org/portal/resource/pt/mdl-21071279.

24. Dekker RL, Lennie TA, Doering LV, Chung ML, Wu JR, Moser DK. Coexisting anxiety and depressive symptoms in patients with heart failure. Eur. J. Cardiovasc Nurs [Internet]. 2014 [acesso em 25 de Janeiro de 2016]; 13(2):168-76. Disponível em: http://pesquisa.bvsalud.org/portal/resource/pt/mdl24408885

25. Michalis L, Polikandrioti M, Goudevenos J, Koutelekos J, Kyristi H, Tzialas D. Factores associated with depression and anxiety of hospitalized patients with heart failure. Hellenic J. Cardiol [Internet]. 2015 [acesso em 20 de Janeiro de 2016]; 56(1):26-35. Disponível em: http://www.ncbi.nlm.nih.gov/ pubmed/25701969.

26. Mehralian H, Salehi S, Moghaddasi J, Amiri M, Rafiei H. The comparison of the effects of education provided by nurses on the quality of life in patients with congestive heart failures (CHF) in usual and home- visit cares in Iran. Global. J. Health Sci [Internet]. 2014 [acesso em 20 de Janeiro de 2016]; 30(4):256-60. Disponível em: http://www.ccsenet.org/ journal/index.php/gjhs/article/view/32987/20267 .

27. González M, Pedrero V. Estrategias de intervención para mejorar la calidad de vida en pacientes con insuficiencia 
cardiaca. Revista Chil. Cardiol [Internet]. 2013 [acesso em 20 de Janeiro de 2016]; 32(2):134-40. Disponível em: http:// pesquisa.bvsalud.org/portal/resource/pt/lil-719581.

28. Silva FVF, Rabelo AC, Silva LF. Processo de enfermagem no conforto do paciente com insuficiência cardíaca no domicílio. Revista Aquichan [Internet]. 2015 [acesso em 15 de abril de 2016]; 15(1):116-28. Disponível em: http://pesquisa.bvsalud. org/portal/resource/pt/lil-749455.

29. Matsuo H, Tsuchihashi M, Kakinoki S, Takechi S, Kinugawa $\mathrm{S}$, Tsutsui H. Home-based disease management program to improve psychological status in patients with heart failure in japan. J-homecare investigators [Internet]. 2013 [acesso em 20 de Janeiro de 2016]; 77(4):926-33. Disponível em: http:// www.ncbi.nlm.nih.gov/pubmed/23502992.

30. Agren S, Evangelista L, Davidson T, Strömberg A. Costeffectiveness of a nurse-led education and psychosocial programme for patients with chronicheart failure and their partners. J. Clin. Nurs [Internet]. 2013 [acesso em 19 de janeiro de 2016]; 22(15-16):2347-53. Disponível em: http:// www.ncbi.nlm.nih.gov/pubmed/23829407. 